\title{
Erratum: Importance of Quantum Tunneling in Vacancy-Hydrogen Complexes in Diamond [Phys. Rev. Lett. 95, 105502 (2005)]
}

\author{
M. J. Shaw, P. R. Briddon, J. P. Goss, M. J. Rayson, A. Kerridge, A. H. Harker, and A. M. Stoneham
} (Received 20 October 2005; published 14 November 2005)

There is an error in some of the data in Table II in our Letter. The symmetrization of the hyperfine parameters for the dynamic $V_{2}-\mathrm{H}^{-}$structure was, by oversight, performed with respect to the wrong symmetry axis leading to incorrect values for $A_{\|}$and $A_{\perp}$. All of the other results reported in the Letter are unaffected by this error. The corrected results are shown in the revised Table I, below.

The correction is noteworthy since it reverses the relative sizes of the parallel and perpendicular components. The corrected figures now agree extremely closely with, and have the same directional characteristics as, the experimental data of Ref. [1] [Ref. 6 in original]. All conclusions in the Letter are unaffected by this numerical change.

[1] C. Glover, M. E. Newton, P. M. Martineau, S. Quinn, and D. J. Twitchen, Phys. Rev. Lett. 92, 135502 (2004).

[2] K. Iakoubovskii and A. Stesmans, Phys. Rev. B 66, 195207 (2002).

TABLE I. The hyperfine parameters $(\mathrm{MHz})$ for the $V-\mathrm{H}^{-}$and dynamical $V_{2}-\mathrm{H}^{-}$complexes. $\mathrm{A}_{\|}$and $\mathrm{A}_{\perp}$ are given where $\|$ refers to the [111] direction. The experimental values of Ref. [1] [Ref. 6 in original] and Ref. [2] [Ref. 8 in original] are listed.

\begin{tabular}{lrr}
\multicolumn{1}{c|}{ System } & $\mathrm{A}_{\|}(\mathrm{MHz})$ & $\mathrm{A}_{\perp}(\mathrm{MHz})$ \\
\hline Static $V-\mathrm{H}^{-}$ & -86.6 & 48.1 \\
Dynamic $V_{2}-\mathrm{H}^{-}$ & 1.2 & 2.0 \\
Expt. Ref. [1] & \pm 1.1 & \pm 2.0 \\
Expt. Ref. [2] & \pm 2.8 & \pm 1.1 \\
\hline \hline
\end{tabular}

